\title{
Why Is the Universe of Sets Not a Set?
}

\author{
Zeynep Soysal
}

\begin{abstract}
According to the iterative conception of sets, standardly formalized by ZFC, there is no set of all sets. But why is there no set of all sets? A simple-minded, though unpopular, "minimal" explanation for why there is no set of all sets is that the supposition that there is contradicts some axioms of ZFC. In this paper, I first explain the core complaint against the minimal explanation, and then argue against the two main alternative answers to the guiding question. I conclude the paper by outlining a close alternative to the minimal explanation, the conception-based explanation, that avoids the core complaint against the minimal explanation.
\end{abstract}

Keywords Actualism · Potentialism · Iterative conception of set · Universe of sets

\section{Introduction}

According to the iterative conception of sets, standardly formalized by ZermeloFraenkel set theory with Choice (henceforth 'ZFC'), there is no set of all sets. Following common usage, let 'the universe of sets' refer to the "totality" of sets in the iterative hierarchy of sets. ${ }^{1}$ Two questions naturally arise: (i) What, if not a set, is the universe of sets? (henceforth 'the what-question') and (ii) Why is the universe of sets not a set? (henceforth 'the why-question'). The why-question will be the guiding question of this paper, and investigating it will require us to look at the what-question. Note that there are equivalent what- and why-questions about other "totalities" that are not members of any sets, such as the "totality" of all ordinals; although I won't directly address those questions in this paper, the discussions that follow could be generalized to apply to them as well.

Zeynep Soysal

Department of Philosophy, Harvard University, 25 Quincy Street, Cambridge, MA 02138

E-mail: soysal@fas.harvard.edu

1 I intend the expression 'the universe of sets' to be neutral with respect to the nature of the "totality" of all sets in the iterative hierarchy. As we will see in this paper, the universe of sets may be a proper class, a plurality, an incomplete totality, a potential hierarchy (in which case there does not actually exist a plurality of all sets), etc. 
Here is a simple-minded response to the why-question:

Minimal Explanation The universe of sets is not a set because the assumption that it is contradicts some axioms of $\mathrm{ZFC}^{2}$

There are a number of different ways to derive a contradiction from the assumption that the universe of sets is a set-e.g. via the Foundation and Pairing axioms, the Separation axiom, the Burali-Forti argument, Cantor's Theorem-and each one generates a more specific instance of the minimal explanation. To have a clear example in mind, let me expand the instance of the minimal explanation generated by the proof from Foundation and Pairing (henceforth 'the Minimal Explanation ${ }_{F, P}$ ') by spelling out how the contradiction is derived: "The universe of sets $V$ is not a set because the assumption that it is contradicts Foundation and Pairing, as follows. If you assume that $V$ is a set, then $\{V\}$ is also a set (by Pairing). Thus $\{V\}$ must have a member that is disjoint from $\{V\}$ (by Foundation). The only member of $\{V\}$ is $V$, hence $V \cap\{V\}=\emptyset$. But $V \in V$ (by definition of $V$ ), so $V \cap\{V\} \neq \emptyset$. Contradiction."

Many philosophers seem to think that the minimal explanation is not a good answer to the why-question. In this paper, I first make precise the core complaint against the minimal explanation $(\$ 2)$, and then argue against the two main alternative answers to the why-question: the size-actualist answer ( 33$)$ and the potentialist answer ( 44$)$. The problems with the alternative answers give us reason to reconsider the minimal explanation as an answer to the why-question. I conclude the paper by outlining a close alternative to the minimal explanation, the conception-based explanation, that avoids the core complaint against the minimal explanation.

\section{The Core Complaint Against the Minimal Explanation}

Philosophers frequently note that one can derive a contradiction from the assumption that there is a set of all sets, but then they simply repeat the why-question. Here is Keith Simmons doing just that:

[W]ith the combinatorial/iterative conception in mind, why can't we "collect together" or "lasso" all the sets in the ZF hierarchy, and form the collection of them all? (Simmons, 2000, 111)

More recently, right after stating the instance of the minimal explanation with Separation, James Studd asks: "What is it about the world that allows some sets to form a set, whilst prohibiting others from doing the same?" (Studd, 2013, 699). It is natural to take such philosophers to think that the minimal explanation doesn't explain why the universe of sets is not a set. Some-like Michael Dummett here concerning the instance of the minimal explanation with Cantor's Theorem-make this thought more explicit:

\footnotetext{
2 The discussion can be generalized to other why-questions by formulating equivalent forms of the minimal explanation. So, for instance, the equivalent "minimal explanation" for the why-question about the "totality" of all ordinals would be: "The totality of all ordinals is not a set because the assumption that it is contradicts some axioms of ZFC."
} 
A mere prohibition leaves the matter a mystery. ... And merely to say, "If you persist in talking about the number of all cardinal numbers, you will run into contradiction," is to wield the big stick, but not to offer an explanation. (Dummett, 1991, 315-316)

However, it is hard to find a clear statement of what exactly is wrong with the minimal explanation as an answer to the why-question. The following two passages contain what I take to come closest to a precise complaint against the minimal explanation.

Right before stating his own potentialist answer to the why-question, Stephen Yablo states:

This [Minimal Explanation ${ }_{\mathrm{F}, \mathrm{P}}$ ] brings what the Russell set is by nature into conflict with a basic fact about sets, viz. well-foundedness. But one may question whether the fact is basic enough. To say that ... [the universal set] cannot exist because it would be ill-founded seems to get things the wrong way around. It is because sets like ... [the universal set] are independently problematic that we are drawn to a requirement that keeps those sets out. (Yablo, 2004, 149)

One way to understand Yablo here is as follows: There is some fact about the universe of sets that explains both why it is not a set and why we adopt Foundation as an axiom (i.e. why "we are drawn to a requirement that keeps those sets out" (ibid.)). So, even though we can derive that the universal set doesn't exist from Foundation, we have to provide that "deeper" fact in explaining why the universal set doesn't exist. That fact, as Yablo later goes on to propose, concerns the "potential" nature of the universe of sets. ${ }^{3}$

In the same vein, James Studd, who also gives a potentialist answer to the whyquestion, suggests that the answer to the why-question ought to appeal to something about sets, and not merely to what can be derived within certain theories:

The derivation of Russell's paradox in Naïve Set Theory demonstrates the logical falsity of the instance of the Naïve Comprehension schema ... . This provides-I am happy to grant-as good an explanation as we should expect for why this theory is inconsistent. However, the question of real interest is not why this instance of Naïve Comprehension yields a contradiction, but why certain sets - in this case, those that lack themselves as elements-are unable to form a set. And this cannot be explained merely by appeal to logical truths.

(Studd, 2013, 700)

I thus propose we understand the core complaint against the minimal explanation as follows. The minimal explanation is a perfectly good answer to the following question: "Why is the universe of sets not as set according to ZFC?" But the minimal explanation fails to provide the deeper reason why these axioms that prohibit the existence of the universal set are there in the first place. So the minimal explanation is not a deep enough explanation of why the universe of sets is not a set; it is not a good answer to the why-question.

\footnotetext{
${ }^{3}$ See for instance Yablo $(2004,152-155)$.
} 
Understanding the core complaint this way also helps make sense of the alternative answers to the why-question. Indeed, most philosophers propose to answer the why-question by appealing to some "deep" fact about the nature of the universe of sets-one that may also provide some independent motivation or explanation for the axioms of set theory that prohibit the existence of the universal set. Their answers naturally go with either of two theories concerning the deep nature of the universe of sets: actualism (more specifically, the Limitation of Size Principle) and potentialism. In what follows, I will argue in turn against the size-actualist and the potentialist answers to the why-question. Of note is that my arguments in this paper aren't directed against actualism or potentialism in general; they are only directed against their respective answers to the why-question. Of course, if the main motivation to endorse either actualism or potentialism is to answer the why-question, then my arguments in this paper should also count against actualism or potentialism. At the beginning of $\S 4$, I will provide some quotes from potentialists that suggest that answering the why-question is a crucial motivation to endorse potentialism; but I will not go further in arguing against the general potentialist thesis in this paper.

\section{Actualism}

For lack of standard terminology, let me use 'actualism' as the name of the general thesis that the universe of sets is some sort of "completed totality." Actualism is thus an answer to my what-question. Proponents of actualism include George Boolos, John Burgess, David Lewis, Gabriel Uzquiano, and arguably Kurt Gödel. Some actualists take a "completed totality" to be a proper class: a well-founded extensional object that is not a set. ${ }^{4}$ Others take it to be a plurality that cannot be "singularized" into one object (either a set or a proper class). ${ }^{5}$

Actualists accept some version of the Limitation of Size Principle, if only because it is derivable in most set theories with proper classes or pluralities: ${ }^{6}$

Limitation of Size Principle A collection, $C$, is not a set if and only if it is of the same size as $V$ (the totality of all sets) or $\Omega$ (the totality of all ordinals).

A number of actualists appeal to the Limitation of Size Principle to justify some of the axioms of ZFC, by stating that an axiom that asserts the existence of sets with a certain property is true if the relevant sets are "small" enough. ${ }^{7}$ Similarly, such actualists use the Limitation of Size Principle to provide an explanation for why the universe of sets is not a set: The universe of sets is not a set because it is "too large" to be a set. Let me call this 'the size-explanation', and actualists who endorse the size-explanation 'size-actualists'. 8

\footnotetext{
4 See for instance Welch (ms), Lewis (1991), and Mayberry (1986).

5 See for instance Boolos (1984), Burgess (2004), and Uzquiano (2003).

6 The derivation requires some version of the axiom of Global Choice. See for instance Linnebo (2010, 162) for the derivation of a limitation of size principle from plural set theory.

7 See for instance Fraenkel et al. (1973). For a thorough study of the history of the Limitation of Size Principle from Cantor to von Neumann and beyond, see Hallett (1984).

8 Size-actualism is defended in Fraenkel et al. (1973) and by Georg Cantor, John Von Neumann, Dmitry Mirimanoff and other Limitation of Size-Theorists, as explained in Hallett (1984). More recently, Welch
} 
My aim in this section is to criticize the size-explanation as an answer to the whyquestion. Many philosophers have already objected to the size-explanation, but I think they did so on the wrong grounds. I will thus first consider the most common objection to the size-explanation, which I call the 'Arbitrary Threshold Objection', and argue that it fails to undermine the size-explanation (\$3.1). The Arbitrary Threshold Objection is often also taken to be a problem for actualism more generally; in $\S 3.1$, I will respond to the objection on behalf of all actualists (including size-actualists). I will then present a different objection to the size-explanation ( 33.2$)$.

\subsection{The Arbitrary Threshold Objection}

Øystein Linnebo formulates the Arbitrary Threshold Objection nicely in (Linnebo, 2010). ${ }^{9}$ According to Linnebo, the actualist is committed to there being a "threshold cardinality beginning at which pluralities are too large to form sets" (Linnebo, 2010, 152). Let us call it ' $\mathrm{T}$ '. He states that the actualist may characterize $\mathrm{T}$ either explicitly as, for instance, the first strongly inaccessible cardinal, or implicitly by using the plurality of all ordinals as a "measuring stick" and understanding T as the cardinality instantiated by the plurality of all ordinals (Linnebo, 2010, 152). He then challenges the actualist as follows:

The main challenge [for the actualist] will be to motivate and defend [T] ... . Why should this particular cardinality mark the threshold? Why not some other cardinality?

... Wherever it has been possible to go on to define larger sets, set theorists have in fact done so. So it remains arbitrary that there should be no sets of this cardinality or some even larger one. (Linnebo, 2010, 152-153) ${ }^{10}$

Linnebo's challenge for the actualist is to explain why $\mathrm{T}$ is not an arbitrary threshold, and to do so in a way that doesn't fly in the face of the practice of set theorists to define sets of larger and larger cardinalities. The Arbitrary Threshold Objection, then, is that the actualist can't meet this challenge.

Let me take up this challenge on behalf of the actualist. My goal is to show that the actualist can coherently maintain the existence of both (i) a distinction between sets and classes (or pluralities that don't form sets), (ii) a non-arbitrary threshold T, and (iii) sets of larger and larger cardinalities.

The first thing to note is that for set theorists, $\kappa$ is a cardinal if and only if $\kappa$ is an ordinal, and for every $\eta<\kappa$, there is no one-to-one correspondence between $\eta$ and $\kappa$ (i.e. $\eta \not \approx \kappa$ ). The cardinality of a given set $A$, written ' $|A|$ ', is in turn defined as the

(ms, 9) assumes some version of the size-explanation. Arguably (and as Linnebo states in Linnebo (2010, 151, fn. 9)) size-actualists also include Burgess (2004) and Lewis (1991). Actualists who aren't sizeactualists include Peter Koellner and Hugh Woodin; they endorse some version of the minimal explanation.

9 I take Linnebo's statement of the Arbitrary Threshold Objection to be the clearest, but others make it too. See for instance Fine (2006, 23), Studd (2013, 700), and Yablo (2004, 152f., 155).

10 See also Linnebo $(2013,206)$ : "To disallow such a set [of all sets] would be to truncate the iterative hierarchy at an arbitrary level." 
least ordinal $\kappa$ such that $A \approx \kappa$ (hence for all $A,|A|$ is a cardinal). ${ }^{11}$ Since all ordinals are sets, crucially, all cardinals and cardinalities are sets.

The actualist accepts these definitions. She then agrees with her challenger that there are sets of larger and larger cardinalities, as is borne out by the practice of set theory. The important point comes now: The actualist doesn't think of $\mathrm{T}$ as a cardinality in the sense above. If she did, the Arbitrary Threshold Objection would go through: How could she maintain both that there are sets of larger and larger cardinalities, and that there is a threshold cardinality above which there are no sets of larger cardinality? The threshold would not only be arbitrary, but inconsistent with her commitments.

The actualist will think of the "size" $\mathrm{T}$ differently. For instance, she may define 'same size' by stating that $A$ has the same size as $B$ if and only if $A \approx B$, and at least partially define 'size' by stating that (a) if $A$ has a cardinality $|A|$, then the size of $A$ is $|A|$, and (b) if $A$ doesn't have a cardinality and has the same size as $V$, then the size of $A$ is T. Now the "location" of T is certainly not arbitrary, because $\mathrm{T}$ is the first size at which the assumption that $A$ has size T implies, on pain of contradiction, that $A$ is not a set. ${ }^{12}$ Moreover, the existence of larger and larger cardinalities doesn't have any bearing on the "location" of T. It is true that for any cardinality $\kappa$, there are sets $A$ of cardinality greater than $\kappa$. It is also true that every cardinality is a size. But it surely doesn't follow from these two facts that for any size S, there are sets of size greater than $\mathrm{S}$.

At this point, opponents of actualism (or size-actualism) could raise a variant arbitrariness complaint: "It would be arbitrary to maintain that for any cardinal $\kappa$, there are sets $A$ of cardinality greater than or equal to $\kappa$, but that for some size $\mathrm{T}$, there are no sets $A$ of size greater than or equal to T." The actualist should respond that there simply are no sets $A$ that have size greater than or equal to $\mathrm{T}$, on pain of contradiction. She may appease her opponents by accepting that there are collections of size greater than T (such as "Super-Classes," collections of size greater than classes that are neither sets nor classes), ${ }^{13}$ in other words, she may (perhaps reluctantly) accept that just as there are larger and larger cardinalities, there are larger and larger sizes. Either way, though, her crucial point is that not all sizes are sizes of sets, on pain of contradiction, and hence that, yet again, nothing about $\mathrm{T}$ has been shown to be arbitrary.

Finally, opponents of actualism may question the actualist's reliance on the distinction between cardinalities and sizes, and the distinction between sets and classes. They may claim that there is a natural kind, so to say, of all things that intuitively count as "collections," that set theory is supposed to be the study of those, and hence that anything that intuitively counts as a "collection" should be treated as a set. From the actualist's perspective, though, this simply mischaracterizes set theory. On the actualist conception, not all things that intuitively count as "collections" are sets-the

\footnotetext{
11 This definition requires the Axiom of Choice. Without Choice, one can use Dana Scott's trick to define $|A|=\{X \mid X$ is of minimal rank s.t. $A \approx X\}$. Since the collection of sets of a given rank is always a set, cardinalities are always sets according to this definition.

12 One can derive a contradiction from the assumptions that $A \approx V$ and that $A$ is a set by using for instance Replacement.

13 Such entities are discussed for instance in Lévy (1976).
} 
universe of sets is one such example. To support her conception, the actualist could assert that we don't have a pre-theoretic conception of the domain of all "collections," that instead our only access to the subject-matter of set theory is through our theories. And according to our theory, not all things that intuitively count as "collections" are sets, on pain of contradiction. That being said, we can still theorize about all collections by supplementing ZFC with a theory of classes (or even Super-Classes... if one wishes). ${ }^{14}$ Hence set theory is compatible with a fully general study of all things that intuitively count as collections - simply not all collections are sets. Once one accepts the actualist position concerning the nature of the universe of sets, there is nothing arbitrary about T: It is exactly where it is supposed to be. We may have other reasons for rejecting actualism, but the actualist's size-explanation remains coherent at least in the face of the Arbitrary Threshold Objection.

\subsection{The Objection from the Explanatory Insignificance of Size}

That being said, I think there is a much better objection against the size-explanation, which I call the 'Objection from the Explanatory Insignificance of Size'. ${ }^{15}$ The Limitation of Size Principle is a generalization about collections that are not sets, but it is not an explanatory generalization. For a given collection $C$, the principle tells us that it cannot be a set if it is of the same size as $V$ (by Replacement). But then our question is simply pushed back from $C$ to $V$ : "Why can't $V$ be a set?" Most arguments that use the Limitation of Size Principle start off with the assumption that $V$ or $\Omega$ is not a set on pain of contradiction, and show that neither can any collection of the same size as $V$ or $\Omega$ be a set. ${ }^{16}$ But we don't have an argument relating the size of $V$ or $\Omega$ to the reason why these collections cannot be sets. The size-explanation doesn't tell us anything beyond the minimal explanation for why $V$ or $\Omega$ are not sets.

An analogy might be useful here. If you scaled me up (that is, if you increased my height while keeping my shape the same), there is a height $\mathrm{H}$ above which my body would collapse under its own weight. Consider the following question: "Why is this particular size $\mathrm{H}$ the threshold above which I would collapse under my own weight?" The answer to this question will appeal to the "square-cube law," namely that if my size (measured in height) is scaled-up by multiplier $m$, then my surface

\footnotetext{
14 For instance, with Von Neumann-Bernays-Gödel (Gödel, 1940), Morse-Kelley (Kelley, 1955) or Lévy-Ackermann set theories (Ackermann, 1956) as theories of classes, or with the formalization by Burgess (2004) as a theory of pluralities.

15 After raising the Arbitrary Threshold Objection, Linnebo goes on to raise another objection to the size-explanation that is perhaps best understood as a version of the Objection from the Explanatory Insignificance of Size. He states: "To probe further, consider the question why there are not more ordinals than ... $[\Omega]$.... According to the view under discussion, the explanation is that ... $[\Omega]$ are too many to form a set, where being too many is defined as being as many as ... [ $\Omega]$. Thus the proposed explanation moves in a tiny circle. The threshold cardinality is what it is because of the cardinality of the plurality of all ordinals, but the cardinality of this plurality is what it is because of the threshold. I conclude that the response fails to make any substantial progress, and that the proposed threshold remains arbitrary" (Linnebo, 2010, 153f.). Here in $\S 3.2$, I aim to clarify this type of objection, disentangle it from the Arbitrary Threshold Objection, and connect it to the minimal explanation.

16 E.g. Cantor's argument for the Aleph Theorem, which states that every cardinal is an aleph (cf. Hallett $(1984,169))$, and other arguments cited in Hallett $(1984,165 f f$.$) .$
} 
area is scaled up by $m^{2}$ and my volume by $m^{3}$. The reason why that particular size $\mathrm{H}$ marks the threshold is that my bone strength is (roughly) proportional to the surface area of the cross section of my bones, but weight is a function of volume, hence after $\mathrm{H}$, my bones won't be able to support my increased weight.

This explanation appeals to size in a satisfactory way: It connects a particular size $\mathrm{H}$ to the event of my body collapsing under its own weight via constraints of physics. In the case of the size of the universe of sets, we have been given no such explanation: Nothing "connects" the size of the universe to its not being able to form a set via constraints of set-theory. The problem with the size-explanation isn't that the size-actualist presupposes that there is this one particular size after which pluralities don't form sets; rather, the problem is that the size-actualist doesn't mention anything about how size in general, and how this particular size in particular, plays a role in explaining why the universe of sets is not a set. The size-explanation doesn't add any explanation for the why-question beyond the minimal explanation. ${ }^{17}$

\section{Potentialism}

For lack of standard terminology, let me use 'potentialism' as the name of the general thesis that the universe of sets is never "completed." More exactly, for the potentialist, necessarily, no matter how many stages of the hierarchy have been formed, it is always possible that there be a further stage containing sets whose members do not form sets in any of the preceding stages. Potentialism is thus another answer to my what-question. Its proponents include Ignacio Jané, Geoffrey Hellman, Øystein Linnebo, Charles Parsons, Augustín Rayo, James Studd, William Tait, Stephen Yablo, and arguably Kit Fine and Ernst Zermelo. ${ }^{18}$

Most (if not all) potentialists take potentialism to provide an explanation for why the universe of sets is not a set: The universe of sets cannot be a set because the universe of sets is never "completed," and only "completed" totalities can form sets. Here Parsons makes clear that potentialism provides an answer to the why-question; he does this in the context of evaluating Georg Cantor's distinction between "definite multiplicities" and "inconsistent multiplicities" (Cantor, 1899):

I suggest interpreting Cantor by means of a modal language with quantifiers, where within a modal operator a quantifier always ranges over a set ... . Then it is not possible that all elements of, say, Russell's class exist, although for any element, it is possible that it exists. (Parsons, 1977, 346) ${ }^{19}$

\footnotetext{
17 In a recent paper, Christopher Menzel argues that the iterative conception of sets is intuitively consistent with the existence of a proper-class sized set of ur-elements, and proposes a modification to Replacement and Powerset to accommodate these "wide" sets (Menzel, 2014). Menzel (2014) may thus be seen as providing an alternative argument for the insignificance of size in explaining why the universe of sets is not a set: The universe of sets is still not a set according to Menzel's modified formalization of the iterative conception, but this has nothing to do with the "size" of the universe of sets.

18 See for instance Jané (1995), Hellman (1989) and Zermelo (1930). The other potentialists are cited below.

19 Parsons also says that the totalities that don't form sets are "merely potential," where "one can distinguish potential from actual being in some way so that it is impossible that all elements of an inconsistent
} 
In the same vein, Linnebo makes clear that he endorses what he takes to be Cantor's answer to the why-question:

On [the potentialist] ... conception, the hierarchy is potential in character and thus intrinsically different from sets, each of which is completed and thus actual rather than potential. This intrinsic difference affords potentialists ... a reason to disallow the disputed set formation.

... [One] attraction of the potentialist conception emerges in connection with the hard question of the conditions under which some objects are eligible to form a set. ... Cantor's thought is that there is an intrinsic difference between multiplicities that form sets and multiplicities that do not, and that this intrinsic difference explains why some but not all multiplicities are eligible for set formation. (Linnebo, 2013, 206f.) ${ }^{20}$

My aim in this section is to criticize the potentialist's explanation as an answer to the why-question. I will first explain potentialism in more detail ( $\S 4.1)$. I will then argue that on each of two ways of understanding the potentialists's modality, their answer adds no explanation for the why-question beyond the minimal explanation $(\S \S 4.2-4.3)$.

\subsection{The Potential Hierarchy}

When we explain the iterative conception of sets, we usually speak as though sets are formed in time. At the beginning we form no sets: $V_{0}=\emptyset$. At the next stage we form the set of all sets formed so far: $V_{1}=\mathscr{P}\left(V_{0}\right)=\{\emptyset\}$. Continuing in this way we obtain $V_{2}=\mathscr{P}\left(\mathscr{P}\left(V_{0}\right)\right), V_{3}=\mathscr{P}\left(\mathscr{P}\left(\mathscr{P}\left(V_{0}\right)\right)\right)$,... After all the finite stages, we form the set of everything that came before, namely $V_{\omega}=\bigcup_{n<\omega} V_{n}$. We can then start taking powersets again, until the next limit stage, $\omega+\omega$, at which we form the set of everything that came before, namely $V_{\omega+\omega}=\bigcup_{\alpha<\omega+\omega} V_{\alpha}, \ldots$ and so on, thereby forming the cumulative hierarchy of sets (Figure 1).

Potentialists take this informal explanation, "replace the language of time and activity with the more bloodless language of potentiality and actuality" (Parsons, 1977, 355), and then formalize it; their aim is to regiment a way of talking about sets that includes claims about possibility, in particular the claim that any sets (any zero or more sets) can form sets. ${ }^{21}$ To this end, they propose formal systems of set theory supplemented by modal quantifiers ' $\diamond \exists$ ' and ' $\square \forall$ ', and either plural logic (Linnebo), second-order logic (Parsons), or additional "backwards-looking" modal

multiplicity should be actual," and then goes on to say that "where there is an essential obstacle to a multiplicity's being collected into a unity, this is due to the fact that in a certain sense the multiplicity does not exist" (Parsons, 1977, 345, my emphasis). So, for Parsons, the fact that the universe of sets is not a set is "due to" its potential nature.

20 In his recent defense of potentialism, Studd similarly takes potentialism to provide an answer to the why-question; he states that potentialism is supposed to answer the "difficult question" I cited above on page 2, namely the question: "What is it about the world that allows some sets to form a set, whilst prohibiting others from doing the same?" (Studd, 2013, 699, emphasis removed).

21 Studd calls this 'the Maximality thesis' (Studd, 2013, 699), Linnebo calls it '(C)' (Linnebo, 2013, 219), and Parsons introduces it in Parsons (1977, 357). 


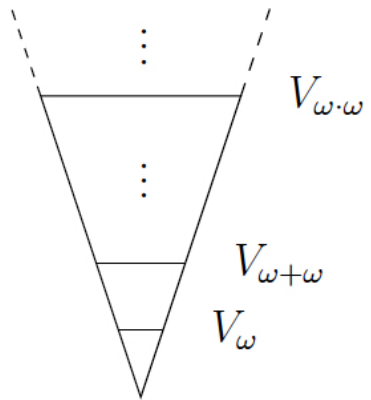

Fig. 1 The cumulative hierarchy of sets.

operators (Studd). Neither formalization adds any new results about sets: A formula $\varphi$ is derivable in ZF if and only if the formula $\varphi^{\diamond}$ that results from replacing each quantifier in $\varphi$ with its corresponding modal quantifier can be proved in modal set theory. ${ }^{22}$ According to potentialists, this shows that "in the context of [their] modal set theory the composite expressions $\square \forall$ and $\diamond \exists$ behave logically just like ordinary quantifiers," and is a reason why "the implicit modalities [they] have postulated in the set-theoretic quantifiers do not surface in ordinary set-theoretic practice" (Linnebo, 2010, 164).

In outline, the potential hierarchy is a structure of possible worlds with an accessibility relation that corresponds to S4.2 (Figure $2^{23}$ ). ${ }^{24}$ Worlds are stages of the set forming process, the domain of each world consists of the sets that have been formed thus far, and the domain of each world accessible from a given world $w_{\alpha}$ is a superset of the domain of $w_{\alpha}$ (Linnebo, 2013, 208). From each world $w_{\alpha}$, there are sets that don't exist at $w_{\alpha}$ but that are possible relative to $w_{\alpha}$, i.e. sets that exist in the domain of a world $w_{\beta}$ which is accessible from $w_{\alpha}$. The overall structure of the potential hierarchy is isomorphic to that of the cumulative $V_{\alpha}$-hierarchy. Moreover, modal set theory and non-modal set theory are "mutually interpretable," in that modalized versions $\mathrm{ZF}^{\diamond}$ of the $\mathrm{ZF}$ axioms are true of the potential hierarchy of sets, ${ }^{25}$ and modal set theory can be interpreted in non-modal set theory (hence is also consistent relative to it). ${ }^{26}$ One way to think about the difference between ordinary set theorists and potentialists is that the latter have "powerful instruments for studying the same

\footnotetext{
22 See for instance Linnebo $(2013,214)$ and Studd $(2013,710)$.

23 Note that, although Figure 2 suggests otherwise, the accessibility relation in S4.2 is not a linear relation; Figure 2 is merely intended to provide a rough visual idea of the potential hierarchy of sets.

24 In particular, Studd's modalization is different from Linnebo's in that it has two basic necessity operators ' $\square_{<}$' and ' $\square_{>}$', and derived ones ' $\square$ ', ' $\square_{\leq}$' and ' $\square_{\geq}$', corresponding respectively to S5 and S4.3 (for the last two). Here I set aside Studd's formalization without loss of generality for my arguments in $\S \S 4.2-4.3$.

25 See for instance Linnebo (2013, 220ff.), Parsons (1983, 318ff.), and Studd (2013, 712ff.).

26 In particular, Linnebo maps his claim (C) (cf. fn. 21) that any sets (any zero or more sets) can form a set onto the claim that for any stage $\alpha$, and any subset of $V_{\alpha}$, there is a later stage $\beta$ such that all later stages $\gamma$ contain a set containing all and only those things in the subset of $V_{\alpha}$ (Linnebo, 2013, 224).
} 


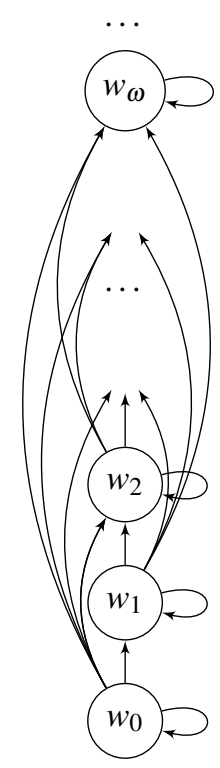

Fig. 2 The potential hierarchy of sets.

subject matter under a finer resolution" (Linnebo, 2013, 206); that is, they "uncover" accessibility relations "between" stages $V_{\alpha}$.

More generally, potentialists see their theses as providing a motivation for the ordinary axioms of set theory; an intermediary, so to speak, between the iterative conception described above and the ZF axioms (or, more exactly, between the iterative conception and the $\mathrm{ZF}^{\diamond}$ axioms, which can in turn be interpreted in ordinary set theory). This intermediary step is supposed to motivate or explain why the axioms hold, as we saw in Yablo's quote in $\S 2$. See also:

[W] hen we confront difficult foundational and conceptual questions concerning set theory, the finer resolution provided by the modal approach can be very valuable. In particular, we will see that the modal approach makes available a very natural motivation for the axioms of ZF set theory. (Linnebo, 2013, $206)^{27}$

Hence potentialists purport to be providing a "deep" account of the nature of the universe of sets in the sense of $\S 2$.

Let me now turn to the potentialist's answer to the why-question. In the potential hierarchy, there is no universal set- there is no world $w_{V}$ at which all sets exist; quantification over sets is always quantification over the domain of a world, but from each world it is always possible to go on to form more sets, and hence there is no

\footnotetext{
27 See also Studd (2013, 698f.) where Studd says of Boolos' stage theory (Boolos, 1971) that it provides a good motivation for the axioms of set theory but is not able to preserve the claim that every sets can form a set nor answer the why-question. Studd then proposes modal set theory as an alternative to stage theory.
} 
"totality" of all sets in any one world. Since there is no "totality" of all sets in this sense, there is also no set of all sets. This is the potentialist's answer to the whyquestion (henceforth 'the potentialist explanation'). ${ }^{28}$

Problems for the potentialist explanation arise once one carefully examines the role and nature of potentialists' notion of modality, which is key in their explanation. All potentialists agree that their modality is not metaphysical modality: Since pure sets and mathematical objects exist necessarily, there is nothing metaphysically potential about the hierarchy of sets. ${ }^{29}$ Instead, they either take the modality as a primitive notion idiosyncratic to mathematics, or interpret it to track set theorists's practice to accept sets of larger and larger cardinalities. ${ }^{30}$ These two options generate two slightly different versions of potentialism. In what follows, I evaluate the potentialist explanation under each option.

\subsection{Primitive and Idiosyncratic Modality}

The first option is to take the potentialist's modality as "mathematical modality," a primitive notion idiosyncratic to mathematics (Parsons, 1983, 327). Linnebo similarly proposes:

Strictly speaking, all we need to assume about the above notion of modality is that it is suited to explicating the iterative conception of sets. The modality must thus be one on which the existence of a set is potential relative to the existence of its elements (in the sense that, when some things exist, it is possible for there to exist a set with precisely these things as elements.) All other details are optional. (Linnebo, 2010, 158)

In this subsection, I will argue that the potentialist explanation for the why-question on this first option for interpreting the potentialist's modality is redundant. I will do so in two steps.

\footnotetext{
28 See for instance Parsons (1977, 345), quoted in fn. 19. Or, for instance, see Linnebo (2010), where Linnebo argues that there is no set of all sets because there is no plurality of all sets, which is because the universe of sets is potential in nature. He thus endorses what he takes to be the view of Yablo $(2004,152)$ : "How can there fail to be a determinate pool of candidates [i.e. a plurality of all sets]? According to Yablo, the answer has to do with the iterative conception of sets. The universe of sets is build up in stages. At each new stage we introduce all the sets that can be formed from the objects available at the preceding stage. But there is no stage at which this process of set formation is complete. At any one stage it is possible to go on and form new and even larger sets. This very suggestive answer needs to be spelled out. I will now propose a way of doing so, based on the idea that the hierarchy of sets is a potential one, not a completed or actual one" (Linnebo, 2010, 155).

29 See for instance Linnebo (2010, 158), Studd (2013, 706), Linnebo (2013, 226), Fine (2006, 31), and Parsons $(1983,328)$.

30 Examples of the former include Linnebo (2013) and Parsons (1977). Linnebo considers the latter option in Linnebo $(2010,159)$. Some potentialists simply propose to forgo specifying the modality: "A full-fledged explanation of the modal notions will have to await another occasion" (Linnebo, 2013, 207). See also Studd: "There is a great deal more to be said about each of these views, but it would take us too far afield to say it here. Rather, safe in the knowledge that taking the tense more seriously than usual need not commit us to taking it literally, I shall continue to elaborate on this view in general, leaving it open (within the bounds of LST) how the modality is to be interpreted" (Studd, 2013, 707).
} 
The first step is to notice that the potentialist's modalities come at a cost: Since ordinary set theorists don't use modal quantifiers, potentialists buy the distance between the axioms (and the why-question) and what explains them at the cost of having to reinterpret the practice of set theorists. Potentialists need to establish a correspondence between the practice of set theory and the "worlds" of the potential hierarchy, and, in particular, specify the sets that are "actual" according to their modality. Linnebo considers this difficulty and proposes two options for the potentialist. The first option is to interpret set theorists to be working "at the world" which contains sets "whose existence follows from our strongest, well-established set theory" (Linnebo, 2010,159 , fn. 21). I will examine this option in $\S 4.3$, as it is embodied in a slightly different version of potentialism. The second option is not to specify any "actual" world but instead to assign set theorists an "external" perspective on the potential hierarchy of sets:

[S]et theorists generally do not regard themselves as located at some particular stage of the process of forming sets but rather take an external view on the entire process. It therefore would be wrong to assign ourselves any particular stage of the process. (Linnebo, 2010, 159)

I will focus on this second option for the remainder of this subsection.

Assume set theorists in their practice take an "external" perspective on the potential hierarchy. Note that potentialists, too, allow themselves such a perspective: In order to prove Infinity ${ }^{\diamond}$, for instance, Linnebo and Studd advocate a "reflection principle," $\varphi^{\diamond} \rightarrow \diamond \varphi$, which is understood as the claim that

... the truth of a claim in 'the model' provided by the potential hierarchy of sets ensures that the claim is possible. For a claim $\varphi$ to be true in this 'model' is for $\varphi$ to be true when all its quantifiers are understood as ranging over all possible sets, including ones not yet formed. But for $\varphi$ to be true when understood in this way is simply for its potentialist translation $\varphi^{\diamond}$ to be true.

(Linnebo, 2013, 222)

But if we are allowed to take such an "external" perspective on the whole potential hierarchy, and even understand our quantifiers as ranging over all possible sets, then the why-question simply resurfaces. Remember that philosophers who reject the minimal explanation are quick to ask the why-question concerning the cumulative hierarchy: "Why can't we 'collect together' or 'lasso' all the sets in the cumulative hierarchy and form a set of all sets?" Presumably, they imagine drawing a circle around Figure 1 and want to know why that doesn't represent a set. But why couldn't we ask the same question with respect to the whole potential hierarchy of sets seen from this "external" perspective? Imagine drawing a circle around Figure 2. Can't we now ask: "Why doesn't that represent a (possibly existing) set?"

To answer the why-question for the cumulative hierarchy, the supporter of the minimal explanation can reply that, strictly speaking, set theory happens "inside" the universe $V$, that although we can "look" at $V$ from the outside this doesn't mean that it is a set, and that in fact $V$ is not a set on pain of inconsistency with set theory. The potentialist isn't satisfied with this answer. But what, besides the claim that modal set theory precludes the existence of a world at which all sets exist, can the potentialist 
offer as an answer to the why-question for the potential hierarchy? She will similarly point out that sets strictly exist "inside" worlds, that although we can (and do) look at the whole hierarchy from the "outside" this doesn't mean that we can form its set, and that modal set theory is designed so that assuming that a universal set is possible entails a contradiction. ${ }^{31}$ So the potentialist will have to provide us with a version of the minimal explanation from within modal set theory, instead of providing the minimal explanation from within ZFC.

This isn't yet a devastating problem for the potentialist. Indeed, potentialists may reply that modal set theory captures some deeper truths about sets than does ZFC, and hence claim that the minimal explanation within modal set theory is more satisfactory than the minimal explanation within ZFC. But this is where the potentialist's primitive and idiosyncratic notion of modality causes trouble. The second step of the argument is thus to note, as we did above in $\S 4.1$, that the potential and iterative hierarchies are isomorphic, and modal and non-modal set theories are mutually interpretable. ${ }^{32}$ This means we cannot get any grip on the potentialist's modality by merely considering the set of true sentences containing ' $\square$ ' and ' $\diamond$ '. If, moreover, we are given no independent grip on potentialist's notion of modality (because we are told it is primitive and idiosyncratic to set theory), then what stops us from simply interpreting the domains of the worlds $w_{\alpha}$ as stages $V_{\alpha}$ defined in ZFC? What exactly is added by the ' $\square$ ' and ' $\diamond$ ' in front of quantifiers? Potentialism on this option starts to look like a notational variant of set theory. And this surely affects its explanatory power: To say that the universe of sets is not a set because it is "potential" in that at any stage, we "can" form more sets in this unspecified and idiosyncratic sense of "can" is not far from giving a dormitive virtue explanation, or saying nothing at all. In other words, simply having unexplained ' $\square$ ' and ' $\diamond$ ' in front of the quantifiers in the minimal explanation doesn't make the potentialist explanation any deeper or more informative than the minimal explanation. The potentialist explanation with this unexplained, primitive and idiosyncratic notion of modality doesn't provide any deeper insight on the why-question than the minimal explanation.

We have just seen that in order to answer the why-question, potentialists commend a costly detour through modal set theory. On the way of incurring the cost just considered, their explanations ultimately rely on a minimal explanation and add nothing to it. I will argue the same for the second way of incurring the cost in $\S 4.3$.

\subsection{Interpreted Modality}

The second option is to interpret the potentialist's modality as somehow tracking the practice of set theorists to accept the existence of larger and larger cardinals. As we saw in $\S 4.2$, Linnebo considers this option in an attempt to re-interpret the practice of set theorists:

\footnotetext{
31 See Linnebo $(2013,222 \mathrm{f}$.) for one example of this kind of "design."

32 One further result deserves mention here besides the one in $\S 4.1$. If $\varphi$ is a formula all of whose quantifiers are modalized, then modal set theory proves that $\varphi, \square \varphi$ and $\diamond \varphi$ are equivalent (see e.g. Studd $(2013,709)$ and Linnebo $(2013,213))$, meaning that it doesn't matter at which world a full modalized formula is evaluated, and which is "another reason why the implicit modalities that I have postulated in the set-theoretic quantifiers do not surface in ordinary set-theoretic practice" (Linnebo, 2010, 164).
} 
As science progresses, we formulate set theories that characterize larger and larger initial segments of the universe of sets. At any one time, precisely those sets are actual whose existence follows from our strongest well-established theory. (Linnebo, 2010, 159, fn. 21) 33 $^{33}$

The version of potentialism associated with this interpretation of the modality is subtly different from the one we considered previously. My criticisms in this subsection will only concern the main idea behind this version of potentialism, so I will keep my exposition at a high level of generality; more detailed accounts can be found in Koellner (ms), Parsons (1974), Rayo and Linnebo (2012) and Tait (2005).

In outline, potentialists of this variety claim that sets exist if and only if they are specified by a theory (call this claim 'Specifiability'). The motivation for Specifiability may be a more constructivist or postulationist metaphysical outlook. Some potentialists put constraints on the kinds of theories that can specify sets in Specifiability. Tait, for instance, mentions the requirement of categoricity:

[W] e may speak about the existence of this or that object in mathematics only when we have specified a consistent and categorical theory in which we speak of such objects. (Tait, 2005, 141)

Others simply let the theories in question be the ones accepted by set theorists.

The second main claim these potentialists endorse is that theories can always be expanded (call this 'Expandability'). Then, Specifiability and Expandability together provide the potentialist with an answer to the why-question: Since sets exist only if they are specified by theories, and since theories can always be expanded, there is no one completed totality of "all sets" which can form a set. In other words, just as it makes no sense to "collect together" all theories, it similarly makes no sense to "collect" together the universe of sets. Here is a quote I take to be voicing this idea:

[The potentialist] view makes it impossible to draw a clean separation between the question of how one might extend one's expressive resources and the question of how many sets exist. By increasing one's expressive resources in the right sort of way, one is led to recognize additional ontology. So insofar as one believes that the process of extending one's expressive resources is essentially open-ended, one should also think that the hierarchy of sets is essentially open-ended - and therefore that there is no definite fact of the matter about what sets there are. (Rayo and Linnebo, 2012, 292)

Once again, there are various ways to understand Expandability. Some philosophers, like Linnebo in the quote above, take theories to be "expandable" in that set theorists define and accept larger and larger cardinals (e.g. Inaccessible, Mahlo, Measurable, Strong, Woodin, Supercompact, etc. $)^{34}$ One problem with this understanding is that it risks making the expandability of theories - and thereby also the nonexistence of a set of all sets-contingent on the particular choices and activities of set theorists. Would there be a universal set if set theorists stopped accepting the exis-

\footnotetext{
33 See also Linnebo (2013, 207f.).

34 See Koellner (2011) for more on the Large Cardinal Hierarchy.
} 
tence of new cardinals? Surely not, since a universal set would still be inconsistent. ${ }^{35}$ A more plausible alternative is to say that theories are "expandable" in that for any recursively enumerable theory $T_{n}$ we can define $T_{n+1}=T_{n}+$ "there is an inaccessible $\kappa$ such that $V_{\kappa} \models T_{n}$," or that any theory of the form ZFC $+\exists \varphi$-cardinals can be "expanded" to ZFC + "There is an inaccessible cardinal $\kappa$ such that $V_{\kappa}$ satisfies that there is a $\varphi$-cardinal." 36

Now that we have a clearer picture of the potentialist explanation under this second option for interpreting the potentialist's modality, I want to raise a problem for it that applies on either way of making precise Specifiability and Expandability. The problem is that Expandability and Specifiability are compatible with the existence of a universal set, hence they cannot explain why there is no set of all sets. Indeed, there are set theories with universal sets (i.e. where it is an axiom that there is a set of all sets) for which the same expandability phenomenon applies: One can define larger and larger cardinals within such theories. ${ }^{37}$ To take a specific example, $\mathrm{NFU}^{+}$(that is, Jensen's version of Quine's NF modified to accommodate urelements, called 'NFU', supplemented by Infinity and Choice) can be expanded as far as one is willing to expand ZFC (Holmes, 2001). ${ }^{38}$ Nothing about those theories conflicts with Specifiability. Thus, since Expandability and Specifiability are compatible with the existence of a universal set, they cannot explain the absence of a universal set.

Let me consider a response on behalf of the potentialist. The potentialist may state: "We are not concerned with set theories like $\mathrm{NFU}^{+}$. We are only interested in set theories that formalize the iterative conception of sets, and hence that have Foundation. $\mathrm{NFU}^{+}$doesn't have Foundation. Our claim is that within well-founded theories, it is their expandability that explains why there is no set of all sets." ${ }^{39}$ The potentialist can state the same response with Separation instead of Foundation, for instance. Without loss of generality, I will only focus on the response with Foundation. I now want to argue that this response is unsuccessful, as it renders the potentialist explanation either once again redundant or needlessly costly.

As we know, if we restrict our attention to theories that formalize the iterative conception - and, in particular, to theories that have Foundation-then we have Minimal Explanation $_{\mathrm{F}, \mathrm{P}}$; we can explain why the universe of sets is not a set with a one-line mathematical argument. Instead, the potentialist wants to add a detour

\footnotetext{
35 There are many other problems facing a radical constructivist view on which the existence of sets depends on whether set theorists define them or think about them. For one, it is widely accepted that such a radical constructivist approach will only sanction a much weaker and non-classical set theory. But actualists and potentialists don't want to be revisionists—as we have seen, they want to "recover" most of the axioms of ZF. So I set aside this kind of revisionist option in this paper. For a recent discussion and summary of the arguments against constructivism, see for instance Incurvati (2012).

36 This way of making sense of expandability fits with Tait (2005) and Parsons (1974).

37 For exposition of set-theories with universal sets, see Forster (1992), Holmes (1998) and Holmes (2001).

$38 \mathrm{NFU}$ is consistent (relative to $\mathrm{PA}$ ) and $\mathrm{NFU}^{+}$is consistent relative to Zermelo set theory with only $\Delta_{0}$-Comprehension (Jensen, 1969). $\mathrm{NFU}^{+}+$the Axiom of Cantorian Sets (which says that all Cantorian sets are strongly Cantorian) proves the existence of inaccessible cardinals, and $n$-Mahlo cardinals for each $n$. A Cantorian set is a set $A$ such that $|A|=\left|\mathscr{P}_{1}(A)\right|$, where $\mathscr{P}_{1}(A)$ is the set of all one-element subsets of $A$, and a set $A$ is strongly Cantorian if the class map $(x \mapsto\{x\})\lceil A$ is a set.

39 Potentialists usually make clear that their focus is on the iterative concept of set, so this kind of response is not implausible for a potentialist. See for instance Linnebo $(2010,144)$.
} 
through Expandability. But this is an unnecessary detour, given that Expandability by itself is compatible with the existence of a universal set; Expandability is an idle wheel in the explanation of why there is no set of all sets. The core problem with the universal set isn't that set theories such as ZFC can be expanded: The core problem is that a universal set would be inconsistent with the assumptions of a theory that formalizes the iterative conception of sets, such as ZFC.

One might object to this argument by denying something like the following principle, which seems to be implicit in it: If a fact $p$ is compatible with $q$ not obtaining, then $p$ cannot explain $q .^{40}$ However, a related worry faces the potentialist explanation whatever stance one takes towards this principle. At this stage of the dialectic, potentialists face two alternative explanations: The first one (i) appeals to Expandability, Specifiability, Foundation and Pairing, the second one (ii) (Minimal Explanation ${ }_{F, P}$ ) appeals to Foundation and Pairing. If the potentialist insists on providing explanation (i), then she incurs costs both because (i) is much more complicated than (ii), but also because (i) contains an extra assumption, Specifiability, that needs to be defended. Indeed, why think that sets are specifiable totalities in the first place? This assumption isn't even part of the iterative conception of sets. The potentialist here buys the explanatory distance at the cost of having to defend a substantial metaphysical assumption about the nature of sets.

\section{The Conception-Based Explanation}

In $\S \S 3-4$, I argued that both the size-explanation and the potentialist explanation rely on the minimal explanation and add nothing to it. I take the problems with these alternative answers to give us reason to reconsider the minimal explanation as an answer to the why-question. But I am also convinced that the core complaint against the minimal explanation stated in $\S 2$ is correct. The why-question asks why the universe of sets is not a set, while the minimal explanation only explains why the universe of sets is not a set according to ZFC; we should thus demand at least some independent reason why the axioms that prohibit the existence of the universal set are there in the first place.

In what follows, I propose a close alternative to the minimal explanation, which I call 'the conception-based explanation'. I argue that the conception-based explanation avoids the core complaint against the minimal explanation at least as well as the size-actualist and potentialist explanations. The conception-based explanation is also more parsimonious than the size-actualist and potentialist explanations, and it doesn't face the problems discussed above in $\S \S 3-4$. I conclude that the conception-based explanation is the best available answer to the why-question.

In $\S 4.1$, I explained the iterative conception of sets. More generally, and in line with common usage, I understand a "conception" of sets to be a way of thinking about sets that is generally accepted or presupposed within some particular mathematical community. The iterative conception of sets, arguably originating in Zermelo (1930), is a way of thinking about sets as forming the iterative hierarchy of sets described in

\footnotetext{
40 In the literature on grounding, people call this principle 'the entailment principle' (Rosen, 2010). Eli Chudnoff (ms) and Jonathan Dancy (2004) are among philosophers who deny this principle.
} 
$\S 4.1$. This way of thinking about sets is at the origin of the why-question; philosophers who ask the why-question assume the iterative conception of sets, as can be seen in the quote from Simmons in $\S 2$ or in the following quote from Linnebo:

By 'set' I mean set as on the iterative conception, according to which sets are "formed" in stages. (Linnebo, 2010, 144) $)^{41}$

Now consider the following response to the why-question:

Conception-Based Explanation The universe of sets is not a set because the supposition that it is contradicts some axioms of ZFC, and these axioms are part of the iterative conception of sets.

Just as the minimal explanation has various instances, so does the conception-based explanation. Here is one such instance, in analogy with Minimal Explanation ${ }_{\mathrm{F}, \mathrm{P}}$ :

Conception-Based Explanation $_{F, P}$ The universe of sets is not a set because the supposition that it is contradicts Foundation and Pairing, and Foundation and Pairing are part of the iterative conception of sets.

What the conception-based explanation adds to the minimal explanation is the claim that the axioms that prohibit the existence of a universal set are part of the iterative conception of sets. In particular, what Conception-Based Explanation ${ }_{F, P}$ adds to Minimal Explanation ${ }_{F, P}$ is the claim that Foundation and Pairing are part of the iterative conception of sets. The conception-based explanation is thus a "deep" explanation in the sense of $\S 2$. It provides an independent reason for why the axioms that prohibit the existence of the universal set are there in the first place: They are simply part of our iterative conception of sets, they are part of what we (who ask the why-question) usually believe or presuppose about sets. If the axioms that prohibit the existence of the universal set are indeed part of the iterative conception of sets, then the conception-based explanation is one we-we who ask the why-question and work with the iterative conception of sets-should be happy with.

What remains to be shown is that at least some axioms that prohibit the existence of a universal set are indeed part of the iterative conception of sets. In other words, one needs to defend either that Foundation and Pairing are part of the iterative conception of sets, or that Separation is part of the iterative conception of sets, etc. But recall that many philosophers — and perhaps most famously Boolos (1971) — have already argued that Foundation and Pairing (and Separation) are part of the iterative conception of sets. Here, for instance, is a summary of the argument for why Pairing is part of the iterative conception of sets. According to the iterative conception of sets, every set appears at some stage. Assume that $a$ and $b$ appear at some stage. Without loss of generality, assume that $b$ appears after $a$. Then the pair set $\{a, b\}$ appears immediately after the stage where $b$ appears, since, according to the iterative conception, that next stage is the stage where all the sets of sets formed so far appears. Pairing follows. ${ }^{42}$

A similar argument can be given for the claim that Foundation is part of the iterative conception of sets. ${ }^{43}$ I will not rehearse it here, but instead note that philosophers

\footnotetext{
41 See also Studd $(2013,698)$.

42 This argument can be found for instance in Maddy (1988, 485).

43 Aside from Boolos (1971), see also for instance Shoenfield (1977, 327).
} 
have often maintained that Foundation is not only part of but essential to the iterative conception of sets. See for instance Parsons and Boolos:

One can state in approximately neutral fashion what is essential to the 'iterative' conception: Sets form a well-founded hierarchy in which the elements of a set precede the set itself. In axiomatic set theory, this idea is most directly expressed by the axiom of foundation, which says that any non-empty set has an ' $\in$-minimal' element. (Parsons, 1977, 335f.)

Whatever tenuous hold on the concepts of set and member were given one by Cantor's definition of "set" and one's ordinary understanding of "element," "set," "collection," etc. is altogether lost if one is to suppose that some sets are members of themselves. (Boolos, 1971, 219)

Thus Foundation and Pairing are part of the iterative conception of sets, as is assumed by Conception-Based Explanation ${ }_{\mathrm{F}, \mathrm{P}}$. Similar arguments can be given for other instances of the conception-based explanation, but it is enough for my purposes here to argue for the assumptions of just one instance of the conception-based explanation.

Let us now examine how the conception-based explanation fares in comparison with the potentialist or size-actualist explanations specifically with respect to the core complaint against the minimal explanation stated in $\S 2$.

Take the potentialist explanation first. I claim that the potentialist explanation for the why-question is deep in the same way as the conception-based explanation is deep. As we saw in $\S 4.1$, potentialists motivate the axioms for modal set theory from the iterative conception of sets, and then provide a minimal explanation from within modal set theory. So their explanation is fundamentally of the same kind as the conception-based explanation: The principles that prohibit the existence of a universal set in the potentialist explanation are put forward as being motivated or justified by the iterative conception of sets. The problem with the potentialist explanation is that it commends a spurious and costly detour through principles that are either unexplained (such as the principles of modal set theory with the idiosyncratic and primitive notion of modality, seen in $\S 4.2$ ) or undefended (such as Specifiability, seen in $\S 4.3$ ). These principles are much less obviously part of the iterative conception of sets than Foundation, Pairing, or Separation - unless of course the principles of modal set theory are mere notational variants of $\mathrm{ZF}$, but in which case nothing is added by the potentialist explanation. ${ }^{44}$ So the conception-based explanation fares at least as well as the potentialist explanation with respect to the core complaint: If one accepts that the potentialist explanation avoids the core complaint, then one should also accept that the conception-based explanation avoids the core complaint. But the conception-based explanation (i) is more parsimonious than the potentialist explanation, (ii) makes no controversial assumptions concerning what is part of the iterative conception of sets, and (iii) doesn't face the problems explained in $\S \S 4.2-4.3$.

Now take the size-explanation. The size-explanation for the why-question appeals to the Limitation of Size Principle, which quite plausibly isn't part of the iterative

\footnotetext{
44 Potentialists themselves might agree with this point. Indeed, Linnebo himself simply assumes Foundation in his modal set theory (Linnebo, 2013).
} 
conception of sets. But the Limitation of Size Principle also stems from a particular conception of sets, arguably originating in Cantor's work, according to which sets are "small" collections. ${ }^{45}$ Once again, the conception-based explanation thus fares at least as well as the size-explanation with respect to the core complaint: The conception-based explanation and the size-explanation both avoid the core complaint by appealing to what is part of our conception of sets. But the conception-based explanation doesn't face the serious Objection from the Explanatory Insignificance of Size raised in $\S 3.2$.

So the conception-based explanation fares at least as well with respect to the core complaint as the size-explanation and the potentialist explanation: The sizeexplanation, the potentialist explanation, and the conception-based explanation all avoid the core complaint against the minimal explanation by appealing to what is part of our conception of sets. But the conception-based explanation is more parsimonious and less problematic than either the size-explanation and the potentialist explanation. The conception-based explanation should thus be preferred to both the size-explanation and the potentialist explanation; it is the best available answer to the why-question.

We saw that everyone in the debate over the why-question presupposes the iterative conception. We also saw that size-actualists and potentialists alike appeal to what is part of our conception of sets in explaining why the universe of sets is not a set. Hence the conception-based explanation provides a satisfactory response to the whyquestion by the lights of everyone involved in the debate. This concludes my case for the superiority of the conception-based explanation over its rivals.

At some point, we may want to ask where the iterative conception itself comes from, and how it, in turn, is justified. The answer to these questions could then also provide us with a better understanding of the nature of the conception-based explanation. I will conclude the paper by providing a roadmap for answering these further questions.

In my view, the iterative conception of sets is part of the (or at least $a$ ) concept of set; it is part of what we-we who ask the why-question and work with the iterative conception - mean by 'set' ${ }^{46}$ On this view, not only are Foundation, Pairing, and Separation part of the iterative conception of sets, they are also part of the concept of set; they are part of what we-we who ask the why-question and work with the iterative conception-mean by 'set'. This view is often at least implicitly shared among participants in the present dispute. For instance, at least on the face of it, Linnebo in the previous quote states that the iterative conception is part of what we mean by 'set'. Also in his last quote above, Boolos seems to be stating that if we know anything about the concepts expressed by 'set' and 'member' - at least the concepts expressed by 'set' and 'member' on Cantor's usage of these expressionsthen we know that sets cannot be members of themselves, which is an elementary consequence of Foundation. See for instance also Burgess concerning Separation:

\footnotetext{
45 For more on the conception of sets based on the Limitation of Size Principle, see for instance Maddy (1988, 484f.) or Hallett (1984).

${ }^{46}$ For simplicity, I assume here that the concept of set is the meaning of our expression 'set'.
} 
However formulated, the assumption of separation is so fundamental to Cantorian thought that it is arguably inappropriate to apply Cantor's word 'set' [Menge] to theories (such as Quine's NF and ML) that do not accept it. In other words, separation may be regarded as a partial explication of the concept of set, indicating what sets are supposed to be like if they exist. (Burgess, 2004, 203)

It is beyond the scope of this paper to argue that the iterative conception-or more specifically that Foundation, Pairing, and Separation-are conceptual truths, especially given that the existence of conceptual truths is a highly disputed philosophical claim. ${ }^{47}$ As I explained above, we also don't need to argue for this in order to show that the conception-based explanation is the best available answer to the why-question. What I do want to point out here, however, is that if the iterative conception of sets is indeed conceptually true, then the conception-based explanation should be understood as a kind of conceptual explanation. And this would show that the conception-based explanation is the best possible explanation for why there is no set of all sets.

Let me explain this with a somewhat simplistic analogy. Consider the question: "Why can't one be a happily married bachelor?" The equivalent "minimal" explanation here would be: "One can't be a happily married bachelor, because one would then be both married and a bachelor, and bachelors are unmarried. Contradiction." Now consider the following conceptual explanation that goes slightly beyond this minimal explanation, in that it makes explicit that the facts to which this minimal explanation appeals are conceptual truths: "One can't be a happily married bachelor because one would then be both married and a bachelor, and bachelors are by definition unmarried (or, it is a conceptual truth that bachelors are unmarried). Contradiction." The conceptual explanation here is clearly more satisfactory than the minimal explanation; it gives some independent reason for why the assumptions that prohibit bachelors from being happily married are there in the first place. It is also clearly a satisfactory answer to the given question: Nothing more is needed to explain why one cannot be a happily married bachelor once one knows the definition of 'bachelor'. Now if the iterative conception - or Foundation, Pairing, and Separation - are conceptually true, then the conception-based explanation should be understood to be of the same kind as this conceptual explanation: The fact that there is no universe of all sets is an elementary consequence of conceptual truths about sets, and that is as good an explanation of why the universe of sets is not a set as we can get.

So if the conception-based explanation is indeed a conceptual explanation, we can see why the conception-based explanation is the best answer to the why-question. Of course, much more needs to be said to defend the view that the iterative conception of sets is conceptually true. The bachelor example above only serves to illustrate the point that a conceptual explanation would be all one could ask for; I don't want to suggest that the mathematical case is quite as simple. In order to defend that the iterative conception of sets is conceptually true, one would need to examine, just for

\footnotetext{
47 There is a long line of arguments against the existence of conceptual or analytic truths and/or of their epistemological consequences, including Quine (1951), Putnam (1962) and the most recent Williamson (2007).
} 
one example, how exactly this view fares with the existence of alternative set theories. I leave the question of how exactly the conceptual explanation would look for future research.

Acknowledgements I would like to thank Olivia Bailey, Neil Barton, Selim Berker, Douglas Blue, Ekaterina Botchkina, Warren Goldfarb, Ned Hall, Jens Kipper, Doug Kremm, Elizabeth Miller, Bernhard Nickel, Charles Parsons, Chris Scambler, Kate Vredenburgh, Stephen Yablo, three anonymous referees for this journal, audiences at Harvard University and at the Kurt Gödel Research Center, and Peter Koellner, especially, for very helpful discussions and comments on various versions of this paper.

\section{References}

Ackermann, W. (1956). Zur Axiomatik der Mengenlehre. Mathematische Annalen 131, 336-345.

Boolos, G. (1971). The iterative conception of set. Journal of Philosophy 68, 215231.

Boolos, G. (1984). To be is to be the value of a variable (or to be some values of some variables). Journal of Philosophy 81, 430-449.

Burgess, J. P. (2004). E pluribus unum: Plural logic and set theory. Philosophia Mathematica 12(3), 193-221.

Cantor, G. (1899). Letter to Dedekind. In J. van Heijenoort (Ed.), From Frege to Gödel: A Source Book in Mathematical Logic, 1879-1931. Cambridge, MA: Harvard University Press.

Chudnoff, E. (ms). Grounding and entailment. Unpublished manuscript.

Dancy, J. (2004). Ethics without Principles. Oxford: Oxford University Press.

Dummett, M. (1991). Frege's Philosophy of Mathematics. Cambridge, MA: Harvard University Press.

Fine, K. (2006). Relatively unrestricted quantification. In A. Rayo and G. Uzquiano (Eds.), Absolute Generality, pp. 20-44. Oxford: Oxford University Press.

Forster, T. E. (1992). Set Theory with a Universal Set. Oxford Logic Guides. Oxford: Clarendon Press.

Fraenkel, A. A., Y. Bar-Hillel, and A. Lévy (1973). Foundations of Set Theory. NorthHolland.

Gödel, K. (1940). The Consistency of the Axiom of Choice and of the Generalized Continuum Hypothesis with the Axioms of Set Theory, Volume 3 of Annals of Mathematical Studies. New Jersey: Princeton University Press.

Hallett, M. (1984). Cantorian Set Theory and Limitation of Size. Oxford Logic Guides. Oxford: Clarendon Press.

Hellman, G. (1989). Mathematics without Numbers. Oxford: Oxford University Press.

Holmes, R. (1998). Elementary Set Theory with a Universal Set, Volume 10. Cahiers du Centre de Logique, Academia, Louvain-la-Neuve.

Holmes, R. (2001). Strong axioms of infinity in NFU. Journal of Symbolic Logic 66(1), 87-116.

Incurvati, L. (2012). How to be a minimalist about sets. Philosophical Studies 159(1), 69-87. 
Jané, I. (1995). The role of the absolute infinite in Cantor's conception of set. Erkenntnis 42(3), 375-402.

Jensen, R. B. (1969). On the consistency of a slight (?) modification of Quine's NF. Synthese 19, 250-263.

Kelley, J. L. (1955). General Topology. New York: Van Nostrand.

Koellner, P. (2011). Independence and large cardinals. In E. N. Zalta (Ed.), The Stanford Encyclopedia of Philosophy (Summer 2011 ed.).

Koellner, P. (ms). Actualism and potentialism. Unpublished manuscript.

Lévy, A. (1976). The role of classes in set theory. In G. H. Müller (Ed.), Sets and Classes: on the Work of Paul Bernays, pp. 173-216. North-Holland.

Lewis, D. (1991). Parts of Classes. Oxford: Blackwell.

Linnebo, Ø. (2010). Pluralities and sets. Journal of Philosophy 107(3), 144-164.

Linnebo, Ø. (2013). The potential hierarchy of sets. Review of Symbolic Logic 6(2), 205-228.

Maddy, P. (1988). Believing the axioms. I. The Journal of Symbolic Logic 53(2), 481-511.

Mayberry, J. (1986). Cantorian set theory and limitation of size. by Michael Hallett. Philosophical Quarterly 36(144), 429-434.

Menzel, C. (2014). Wide sets, ZFCU, and the iterative conception. Journal of Philosophy 111(2), 57-83.

Parsons, C. (1974). Sets and classes. Noûs 8(1), 1-12.

Parsons, C. (1977). What is the iterative conception of set? In R. E. Butt and J. Hintikka (Eds.), Proceedings of the 5th International Congress of Logic, Methodology and Philosophy of Science 1975, Part I: Logic, Foundations of Mathematics, and Computability Theory, pp. 335-367. Reidel.

Parsons, C. (1983). Sets and modality. In Mathematics in Philosophy, pp. 298-341. Cornell University Press.

Putnam, H. (1962). The analytic and the synthetic. In H. Feigl and G. Maxwell (Eds.), Scientific Explanation, Space, and Time. Minnesota Studies in the Philosophy of Science, Volume 3, pp. 358-397. University of Minnesota Press.

Quine, W. V. O. (1951). Two dogmas of empiricism. Philosophical Review 60, 2043.

Rayo, A. and Ø. Linnebo (2012). Hierarchies ontological and ideological. Mind 121(482), 269-308.

Rosen, G. (2010). Metaphysical dependence: Grounding and reduction. In B. Hale and A. Hoffmann (Eds.), Modality: Metaphysics, Logic, and Epistemology, pp. 109-136. Oxford: Oxford University Press.

Shoenfield, J. R. (1977). Axioms of set theory. In J. Barwise (Ed.), Handbook of Mathematical Logic, pp. 321-344. North-Holland.

Simmons, K. (2000). Sets, classes and extensions: A singularity approach to Russell's paradox. Philosophical Studies 100(2), 109-149.

Studd, J. (2013). The iterative conception of set. Journal of Philosophical Logic 42(5), 695-725.

Tait, W. (2005). Constructing cardinals from below. In The Provenance of Pure Reason, Chapter 6, pp. 133-154. Oxford: Oxford University Press. 
Uzquiano, G. (2003). Plural quantification and classes. Philosophia Mathematica 11(1), 67-81.

Welch, P. (ms). Conceptual realism: sets and classes. Unpublished manuscript. Williamson, T. (2007). The Philosophy of Philosophy. Malden, MA: Blackwell.

Yablo, S. (2004). Circularity and paradox. In V. F. H. Thomas Bolander and S. A. Pedersen (Eds.), Self-Reference. CSLI.

Zermelo, E. (1930). Über Grenzzahlen und Mengenbereiche. Fundamenta Mathematica 16, 29-47. 